\title{
Comparative in vivo bioequivalence and in vitro dissolution of two valproic acid sustained-release formulations
}

\author{
Akira Fujii' \\ Norio Yasui-Furukori' \\ Taku Nakagami ${ }^{1,3}$ \\ Takenori Niioka ${ }^{4}$ \\ Manabu Saito',2 \\ Yasushi Sato ${ }^{1,2}$ \\ Sunao Kaneko' \\ 'Department of Neuropsychiatry, \\ Hirosaki University School \\ of Medicine, Hirosaki, Japan; \\ ${ }^{2}$ Department of Psychiatry, \\ Hirosaki-Aiseikai Hospital, \\ Hirosaki, Japan; ${ }^{3}$ Department \\ of Neuropsychiatry, Ohdate City \\ Hospital, Ohdate, Akita, Japan; \\ ${ }^{4}$ Department of Pharmacy, Hirosaki \\ University Hospital, Hirosaki, Japan
}

Correspondence: Norio Yasui-Furukori Department of Neuropsychiatry, Hirosaki University School of Medicine, Hirosaki 036-8562, Japan

Tel +8I 172395352

Fax +81172395352

Email yasufuru@cc.hirosaki-u.ac.jp
Objective: A study was conducted to establish the bioequivalence between different sustained-release formulations of valproic acid (Depakene R and Selenica R), which were developed in Japan.

Materials and methods: The clinical investigation was designed in a randomized, crossover fashion with a single dose given to 12 healthy subjects. The subjects were administered a single $600 \mathrm{mg}$ dose of valproic acid in one of two formulations. Serial venous blood samples were obtained over 72 hours after each administration to measure valproic acid in serum by enzyme immunoassay (EIA). In addition, a dissolution test was performed. Each sample was analyzed by an high-performance liquid chromatography to determine the dissolution rate of valproic acid.

Results: No difference in maximum concentration or area under the curve was found between the two formulations. The time to maximum concentration of the new formation was significantly delayed compared with the conventional formulation $(10.8 \pm 1.7$ versus $17.6 \pm$ 1.8 hours, $\mathrm{p}<0.001)$. Apparent clearance or elimination half-life did not differ between the two formulations. An in vitro dissolution study showed that Depakene R was significantly more dissoluble than Selenica R.

Conclusion: Based on the results, the present study demonstrated a significant difference between the two sustained-release formulations in the absorption profile, and also demonstrated that the bioavailability of valproic acid in the two formulations was similar but absorption speed (lag time) was very different.

Keywords: bioequivalence, sustained-release, valproic acid, Depakene R, Selenica R

\section{Introduction}

Successful long-term treatment of patients with epilepsy requires selection of an appropriate antiepileptic regimen, optimal dosing and patient compliance (Pugh and Garnett 1991; Buck et al 1997). Sustained-release formulations of antiepileptic drugs can be very helpful in achieving treatment objectives. Stable serum levels without marked peak-to-trough fluctuations, reduced frequency of dosing, and the possibility of dosing flexibility may all improve compliance, patient satisfaction and, ultimately, quality of life (Bialer 1992; Cramer et al 1995; Pellock et al 2004).

Valproic acid has been widely used in the last decade and is now considered a relatively safe and effective anticonvulsant agent (Perucca 2002). Recently, several investigations have proposed its use in the treatment of anxiety, alcoholism, and mood disorders (Bowden and Singh 2005; Nasrallah et al 2006). Valproic acid is characterized by dose-limited absorption, nonlinear plasma protein binding, and multiple metabolic pathways of elimination (Gugler and von Unruh 1980; Zaccara et al 1988; Perucca 2002). Once absorbed, valproic acid is largely bound to plasma 
proteins and has a relatively small volume of distribution. Its concentration in cerebral spinal fluid is approximately one-tenth that in plasma and is directly correlated with the concentration found in tears. At therapeutic doses, valproic acid's half-life varies from 10 to 20 hours in adults, while it is significantly shorter ( 6 to 9 hours) in children (Zaccara et al 1988; Perucca 2002). Valproic acid undergoes extensive liver metabolism. Numerous metabolites have been positively identified and there is reasonable evidence that several of them contribute to its pharmacological actions or toxicity (Zaccra et al 1988; Eadie 1991).

Valproic acid is available in different dosage forms for parenteral and oral use. All available oral formulations are almost completely bioavailable, but they differ in dissolution characteristics and absorption rates. The sustained-release formulation (Depakene R) can therefore be given once or twice daily (Doughty et al 2003; Genton 2005). The tablet core consists of a matrix structure that is covered with the sustained-released membrane. The elution of valproic acid is controlled as the substance passes through the core of the matrix structure and, further, through the sustained-released membrane.

Recently another sustained-release formulation containing $200 \mathrm{mg}$ valproic acid (Selenica R) has been developed and is available in Japan (Fujisaki et al 2006). Selenica R has a double-coating system that provides a mechanism that is different from the conventional sustained-release formulation. Preclinical studies with healthy volunteers showed there are different pharmacokinetic properties between this new formulation and the conventional formulation (Pers comm.; Nikken Chemical Co., Tokyo, Japan). However, there is no information available on absorption profiles of these two formulations when directly compared. We therefore compared the pharmacokinetic parameters of the two valproic acid formulations in healthy subjects. A comparative study of the dissolution profiles between the two tablets was also carried out.

\section{Methods}

\section{In vitro dissolution test}

A dissolution test was performed according to the procedure described in the JP Dissolution Test (paddle and beads method) (The Japanese Pharmacopeia 15th Edition, Japanese Ministry of Health, Labour and Welfare, http://www.mhlw. go.jp/topics/bukyoku/iyaku/yakkyoku/dl/060407-1.pdf). The paddle rotation speed was maintained at $50 \mathrm{rpm}$ at $37{ }^{\circ} \mathrm{C}$. Release test was carried out in $900 \mathrm{ml}$ of $\mathrm{pH} 6.8$ phosphate buffer $(0.05 \mathrm{~mol} / \mathrm{L})$ using a dissolution tester. Samples of
$10 \mathrm{ml}$ were withdrawn at predetermined time intervals and replaced with the same volume of fresh buffer. Each sample solution was analyzed by high-performance liquid chromatography (HPLC) to determine the dissolution rate of valproic acid as described by Fujisaki and colleagues (2006). An AUV absorption spectrometer was used as the HPLC detector at a detection wavelength of $210 \mathrm{~nm}$. The HPLC column was an Inertsil ODS stainless steel column with an inside diameter of $4.0 \mathrm{bmm}$ and a length of $25 \mathrm{~cm}$ (GL Science, Ins., Tokyo, Japan). The analysis was performed at $40{ }^{\circ} \mathrm{C}$. The mobile phase was $0.02 \mathrm{M}$ phosphate buffer ( $\mathrm{pH} 3.0)$ and acetonitrile $(60 ; 40, \mathrm{v} / \mathrm{v})$.

\section{In vivo bioequivalence study Subjects}

Twelve healthy Japanese volunteers ( 9 males, 3 females $)$ were enrolled in this study. Their mean age $\pm \mathrm{SD}$ and age range were $28.8 \pm 4.8$ and 23-36 years, respectively and mean body weight was $62.3 \pm 13.1$ with a range of $43-95 \mathrm{~kg}$.

\section{Study design}

The study protocol was approved by the Ethics Committee of Hirosaki University School of Medicine. It was a randomized, two-treatment, two-period, crossover investigation with a washout phase of 4 weeks between the two study periods. The treatments consisted of a single oral $600 \mathrm{mg}$ dose with $200 \mathrm{ml}$ water. $600 \mathrm{mg}$ of different sustained-release valproic acid formulations (three $200 \mathrm{mg}$ tablets of Depakene R or Selenica R) were administered in a randomized crossover fashion with a washout period of at least 4-weeks. Six volunteers within each group were allocated to either of 2 different drug sequences: Depakene R-Selenica R and Selenica R-Depakene R. No meal was allowed until 4 hours after the dosing (1 pm). Fluid intake was also standard, and no alcohol, tea, coffee, and cola were allowed during the periods of confinement. Study participants were asked to refrain from the use of all drugs, including over-the-counter medications, for at least 2 weeks before the first administration, as well as during the entire study. Blood samples $(5 \mathrm{ml}$ each) for determination of valproic acid were collected into heparinized tubes just before and $2,4,6,8,10,12,14,16,18,24,36,48,60$, and 72 hours after the administration of valproic acid. Samples collected in the heparinized tubes were gently inverted several times. At the same time as blood sampling, blood pressure, heart rate, and self-reported adverse effects were monitored. Group assignment and the drug administration schedule were kept sealed until the blood samples were analyzed. 


\section{Bioanalytical method}

Serum concentrations of valproic acid were quantified with enzyme immunoassay (EIA). The detection limit was $1.0 \mu \mathrm{g} / \mathrm{ml}$. The inter- and intra-assay CVs for valproic acid were less than $5.5 \%$ for all quality control concentrations.

\section{In vivo pharmacokinetic parameters}

Pharmacokinetic parameters were determined by noncompartment model with WinNonlin software (Pharsight Co.,Cary, NC, USA). Apparent volume of distribution $(\mathrm{Vd} / \mathrm{F})$, absorption constant $(\mathrm{Ka})$, elimination constant $(\mathrm{Ke})$, and lag time (Tlag) were calculated as first parameters. Area under the drug concentration-time curve (AUC), apparent clearance $(\mathrm{CL} / \mathrm{F})$, time to peak concentration $\left(\mathrm{T}_{\max }\right)$, peak concentration $\left(\mathrm{C}_{\max }\right)$, peak concentration at steady state $\left(\mathrm{Css}_{\max }\right)$ and minimum concentration at steady state $\left(\mathrm{Css}_{\min }\right)$ were estimated as second parameters.

\section{Statistical analysis}

Paired t-test was used for the comparison of the pharmacokinetic parameters between the two phases, ie, Depakene $\mathrm{R}$ and Selenica $\mathrm{R}$. Two-way analysis of variance was used for sequence effects. Two-way ANOVA was used for comparison of the in vitro dissolution study. Post hoc analyses were done using Bonferroni's correction with paired t-test.

A $p$ value of 0.05 or less was regarded as significant. When the calculated $90 \%$ confidence intervals with logarithmic transformation of pharmacokinetic data $\left(\mathrm{C}_{\max }\right.$ and AUC) fell within $80 \%-125 \%$ for the ratio of the product average, we regarded the product as bioequivalent. SPSS 13.0J for Windows (SPSS Japan Inc., Tokyo, Japan) was used for these statistical analyses.

\section{Results and discussion In vitro drug release study}

Dissolution curves are shown in Figure 1. All the Depakene $\mathrm{R}$ tablets were detectable after 3 hours, while it took 7 hours to detect all of the Selenica R tablets. Two-way ANOVA showed significant difference in dissolution curves between the two formulations $(\mathrm{p}<0.001)$. Dissolved valproic acid concentrations for Depakene $\mathrm{R}$ were significantly greater than those for Selenica $\mathrm{R}$ from 7 to 24 hours.

These findings suggested the different mechanisms of sustained release between Depakene R and Selenica R, ie, the elution of valproic acid from Depakene $\mathrm{R}$ is controlled in such a way that the substance passes through the core matrix structure and further passes through the sustainedrelease membrane, while Selenica $R$ elutes through a mixed

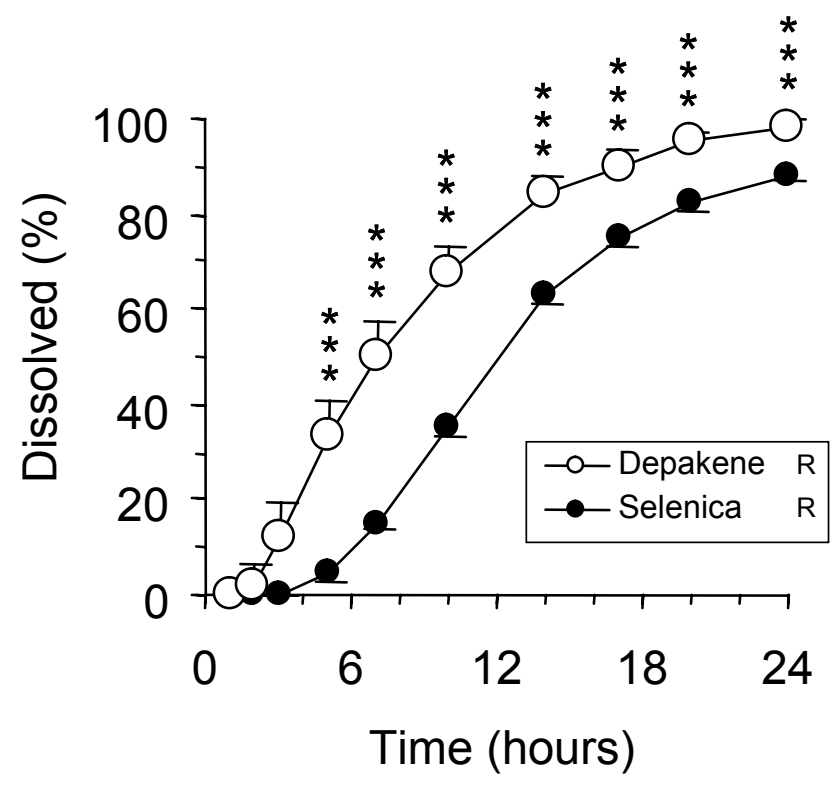

Figure I Dissolution profiles of Depakene R and Selenica R. Error bars indicate standard errors. Open and solid circles are the data for Depakene R and Selenica R, respectively.

Notes: ${ }^{*} p<0.05,{ }^{*} *_{p}<0.01, *^{*} *_{p}<0.001$.

membrane composed of ethylcellulose and methacrylate copolymer-L (Fijisaki et al 2006) (Figure 2).

\section{In vivo bioequivalence study}

Serum concentration-time curves of valproic acid after a single oral dose of Depakene R tablets and Selenica R tablets are shown in Figure 3, and their pharmacokinetic parameters including direct (Table 1) and simulated (Table 2) single oral dose data are summarized. There were no severe adverse events during the study. Two subjects had mild sleepiness from 6 hours to 12 hours for both phases. No difference in blood pressure or heart rate was observed between the two formulations.

There were no differences in $\mathrm{C}_{\max }$ or total AUC between the new formulation (Selenica $\mathrm{R}$ ) and conventional formulation (Depakene R). Bioequivalence analyses showed that $90 \%$ confidence interval of log-transformed $\mathrm{C}_{\max }$ and total AUC between the two formulations were $92 \%-106 \%$ and $101 \%-111 \%$, respectively.

Lag time and $\mathrm{t}_{\max }$ of Selenica $\mathrm{R}$ were significantly delayed in comparison with those of Depakene $\mathrm{R}(7.1 \pm 0.8$ versus $1.7 \pm$ 0.6 hours, $\mathrm{p}<0.001$ for lag time, $17.6 \pm 1.8$ versus $10.8 \pm$ 1.7 hours, $\mathrm{p}<0.001$ for $\mathrm{t}_{\max }$ ). However, no difference was found in $\mathrm{Ka}$. Other parameters, such as $\mathrm{Vd} / \mathrm{F}, \mathrm{Ke}$, and $\mathrm{CL}$, did not differ between the two formulations. $\mathrm{Css}_{\max }$ and Css $\mathrm{min}_{\min }$ simulated by single-oral pharmacokinetic parameters were $63.5 \pm$ 17.7 and $35.1 \pm 10.4 \mu \mathrm{g} / \mathrm{ml}$ for Depakene R and $64.3 \pm 16.0$ and $40.8 \pm 13.1 \mu \mathrm{g} / \mathrm{ml}$ for Selenica R. Css $_{\text {min }}$ of Selenica R was significantly higher than that of Depakene $\mathrm{R}(\mathrm{p}<0.05)$, but the 


\section{Depakene R}

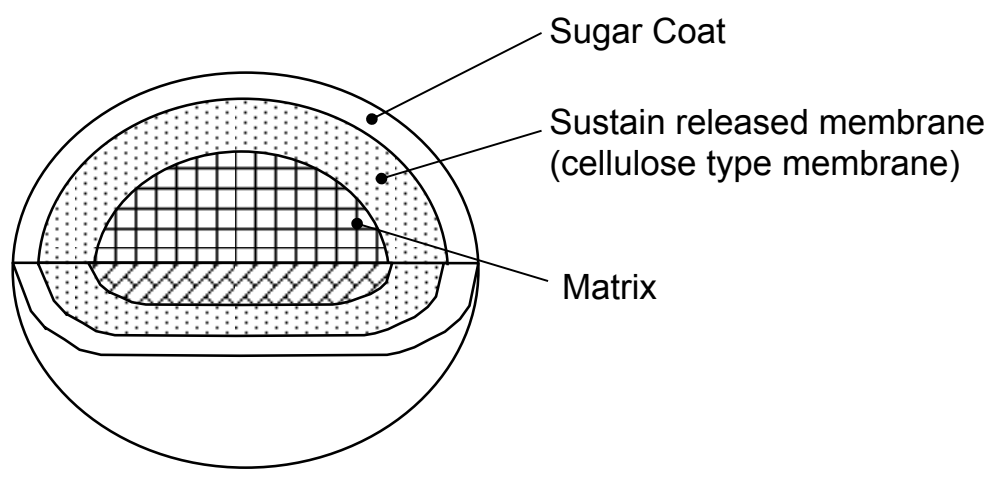

\section{Selenica R}

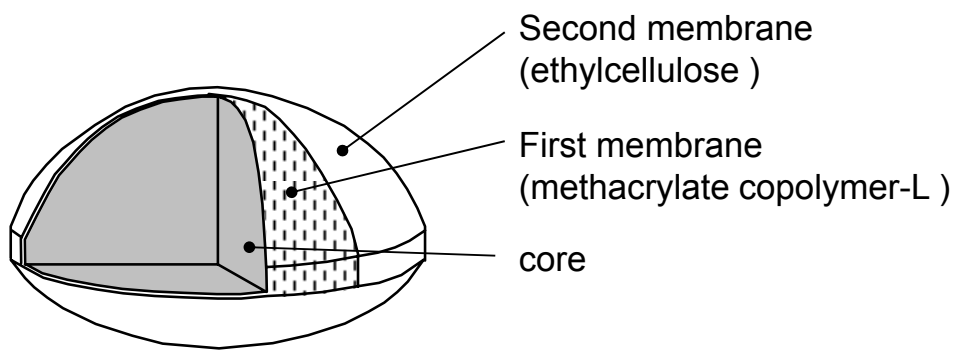

Figure 2 Tablet characteristics for the slow-release preparations of Depakene R and Selenica R.

difference in fluctuating drug concentration $\left(\mathrm{Css}_{\max }-\mathrm{Css}_{\min }\right)$ did not differ between Selenica R and Depakene R. There were no sequence effects on the pharmacokinetic parameters.

The results of this study showed no differences in $\mathrm{C}_{\max }$ or AUC of valproic acid between new and conventional formulations. The $90 \%$ confidence interval of logtransformed $\mathrm{C}_{\max }$ and total AUC between the two formulations fell within $80 \%-125 \%$, suggesting that Selenica $\mathrm{R}$ is bioequivalent to Depakene R according to US Food and Drug Administration Guidance for Industry (see http://www.fda.
gov/CDER/guidance/3616fnl.htm). Therefore, it appears that blood concentrations that cause side effect are comparable between the two formulations.

However, absorption speed, such as lag time and hence $\mathrm{t}_{\max }$, were very different between the two formulations in the single oral dose study. This is supported by the in vitro dissolution study which demonstrated that Depakene $\mathrm{R}$ dissolved earlier than Selenica R. These findings suggest that valproic acid dissolved from Depakene $\mathrm{R}$ appears earlier in the blood and brain than that from Selenica R.

Table I Pharmacokinetic parameters of valproate in healthy subjects $(n=12)$ after single oral doses of valproate $(600 \mathrm{mg})$ in two sustained-release formulations calculated from direct data

\begin{tabular}{llll}
\hline Parameters & Depakene $\mathbf{R}$ & Selenica $\mathbf{R}$ & Significance \\
\hline $\mathrm{C}_{\text {max }}(\mu \mathrm{g} / \mathrm{ml})$ & $42.4 \pm 10.7$ & $40.9 \pm 8.4$ & $\mathrm{NS}$ \\
$\mathrm{T}_{\max }(\mathrm{hr})$ & $11.1 \pm 1.3$ & $16.0 \pm 1.6$ & $\mathrm{P}<0.00 \mathrm{I}$ \\
$\mathrm{AUC}(0-48)(\mathrm{hr} * \mu \mathrm{g} / \mathrm{mL})$ & $1361 \pm 346$ & $1458 \pm 342$ & $\mathrm{NS}$ \\
$\mathrm{AUC}(0-\infty)(\mathrm{hr} \mu \mathrm{g} / \mathrm{mL})$ & $1408 \pm 403$ & $1536 \pm 392$ & $\mathrm{NS}$ \\
Elimination half-life $(\mathrm{hr})$ & $12.9 \pm 2.6$ & $12.7 \pm 2.9$ & $\mathrm{NS}$ \\
\hline
\end{tabular}

Note: Data are shown as mean \pm SD for pharmacokinetic parameters.

Abbreviations: $C_{\text {max }}$, peak concentration; $t_{\text {max }}$, time to peak concentration in serum; $A U C(0-48)$, are under plasma concentration-time curve ( $\left.A U C\right)$ from 0 to 48 hours; $A U C$ $(0-\infty)$, AUC from 0 to infinity; NS, not significant; SD, standard deviation. 
Table 2 Estimated pharmacokinetic parameters in subjects receiving a single oral dose of valproate in two sustained-release formulations $(n=12)$

\begin{tabular}{llll}
\hline Parameters & Depakene R & Selenica R & Significance \\
\hline $\mathrm{Vd} / \mathrm{F}(\mathrm{L})$ & $9.5 \pm 2.9$ & $8.7 \pm 1.9$ & $\mathrm{NS}$ \\
$\mathrm{Ka}(\mathrm{I} / \mathrm{hr})$ & $0.21 \pm 0.08$ & $0.17 \pm 0.07$ & $\mathrm{NS}$ \\
$\mathrm{Ke}(\mathrm{I} / \mathrm{hr})$ & $0.056 \pm 0.012$ & $0.058 \pm 0.014$ & $\mathrm{NS}$ \\
$\mathrm{T}$ lag $(\mathrm{hr})$ & $1.7 \pm 0.6$ & $7.2 \pm 0.8$ & $\mathrm{P}<0.001$ \\
$\mathrm{AUC}(\mathrm{hr} * \mathrm{mg} / \mathrm{mL})$ & $1241 \pm 329$ & $1300 \pm 317$ & $\mathrm{NS}$ \\
$\mathrm{CL} / \mathrm{F}(\mathrm{L} / \mathrm{hr})$ & $0.53 \pm 0.17$ & $0.49 \pm 0.13$ & $\mathrm{NS}$ \\
$\mathrm{T}_{\max }(\mathrm{hr})$ & $10.8 \pm 1.6$ & $17.6 \pm 1.7$ & $\mathrm{P}<0.001$ \\
$\mathrm{C}_{\max }(\mathrm{mg} / \mathrm{ml})$ & $40.2 \pm 10.5$ & $39.3 \pm 8.4$ & $\mathrm{NS}$ \\
$\mathrm{Css}_{\max }(\mathrm{mg} / \mathrm{ml})$ & $63.5 \pm 16.9$ & $64.3 \pm 15.3$ & $\mathrm{NS}$ \\
$\mathrm{Css}_{\min }(\mathrm{mg} / \mathrm{ml})$ & $35.1 \pm 10.0$ & $40.8 \pm 12.5$ & $\mathrm{P}<0.05$ \\
\hline
\end{tabular}

Note: Data are shown as mean \pm SD for pharmacokinetic parameters.

Abbreviations: Vd/F, apparent volume of distribution; Ka, absorption constant; Ke, elimination constant; Tlag, lag time; $\mathrm{AUC}$ (0- $\infty$ ), $\mathrm{AUC}$ from 0 to infinity; $\mathrm{Cl} / \mathrm{F}$, apparent total clearance; $t_{\max }$, time to peak concentration in serum; $C_{\max }$, peak concentration; $\mathrm{Css}_{\max }$, peak concentration at steady state; $\mathrm{Css}_{\min }$, trough concentration at steady state.

On the other hand, no changes in physiological and mental status in our subjects were observed between Selenica R treatment and Depakene R treatment in this study. This is explained by similar exposure (AUC) and/or $\mathrm{C}_{\max }$ in the two formulations. Because therapeutic antiepileptic effects or mood-stabilizing effects are associated with exposure to valproic acid, when a sustained-release preparation is switched to another formulation, inconsistencies in pharmacokinetic parameters, such as lag time and $t_{\max }$, do not necessarily mean alteration in clinical response. This was confirmed by our previous study (Yasui-Furukori et al 2007).

\section{Conclusion}

The present study demonstrated that the bioavailability of valproic acid in two formulations was similar but absorption time was different because of the different dissolution profiles.

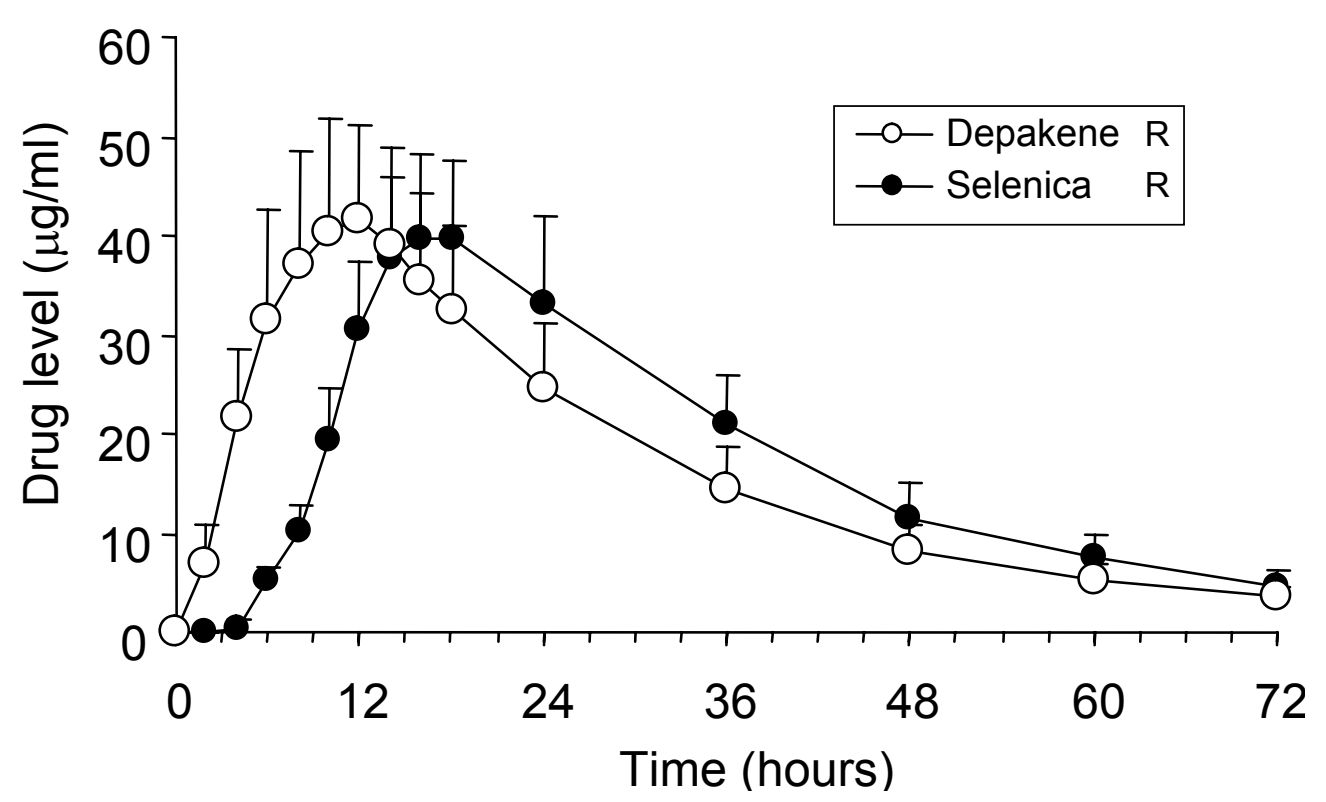

Figure 3 Serum concentration-time curves of valproic acid after single oral doses of Depakene R and Selenica R. Error bars indicate standard errors. Open and solid circles are the data for Depakene $\mathrm{R}$ and Selenica $\mathrm{R}$, respectively. Notes: ${ }^{*} p<0.05$, **p $<0.01$, *** $p<0.001$. 


\section{Acknowledgments}

None of the authors and their institutes have any conflicts of interest. This study was supported by a grant from the Hirosaki Research Institute for Neurosciences.

\section{References}

Bialer M. 1992. Pharmacokinetic evaluation of sustained release formulations of antiepileptic drugs. Clinical implications. Clin Pharmacokinet, 22:11-21.

Bowden CL, Singh V. 2005. Valproate in bipolar disorder: 2000 onwards. Acta Psychiatr Scand Suppl, 426:13-20.

Buck D, Jacoby A, Baker GA, et al. 1997. Factors influencing compliance with antiepileptic drug regimes. Seizure, 6:87-93.

Cramer J, Vachon L, Desforges C, et al. 1995. Dose frequency and dose interval compliance with multiple antiepileptic medications during a controlled clinical trial. Epilepsia, 36:1111-7.

Doughty J, Baker GA, Jacoby A, et al. 2003. Compliance and satisfaction with switching from an immediate-release to sustained-release formulation of valproate in people with epilepsy. Epilepsy Behav, 4:710-6.

Eadie MJ. 1991. Formation of active metabolites of anticonvulsant drugs. A review of their pharmacokinetic and therapeutic significance. Clin Pharmacokinet, 21:27-41.
Fujisaki Y, Tsukune T, Funyu M, et al. 2006. Development of sustained-release tablets containing sodium valproate: in vitro and in vivo correlation. Drug Dev Ind Pharm, 32:207-17.

Genton P. 2005. Progress in pharmaceutical development presentation with improved pharmacokinetics: a new formulation for valproate. Acta Neurol Scand Suppl, 182:26-32.

Gugler G, von Unruh GE. 1980. Clinical pharmacokinetics of valproic acid. Clin Pharmacokinet, 5:67-83.

Nasrallah HA, Ketter TA, Kalali AH. 2006. Carbamazepine and valproate for the treatment of bipolar disorder: a review of the literature. $J$ Affect Disord, 95:69-78.

Pellock JM, Smmith MC, Cloyd JC, et al. 2004. Extended-release formulations: simplifying strategies in the management of antiepileptic drug therapy. Epilepsy Behav, 5:301-7.

Perucca E. 2002. Pharmacological and therapeutic properties of valproate: a summary after 35 years of clinical experience. CNS Drugs, 16:695-714.

Pugh CB, Garnett WR. 1991. Current issues in the treatment of epilepsy. Clin Pharm, 10:335-58.

Yasui-Furukori N, Saito M, Nakagami T, et al. 2007. Different serum concentrations of steady-state valproic acid in two sustained-release formulations. Psychiatry Clin Neurosci, 61:308-12.

Zaccara G, Messori A, Moroni F. 1988. Clinical pharmacokinetics of valproic acid-1988. Clin Pharmacokinet, 15:367-89. 